Relato de Caso / Case Report

\title{
Uso de contraceptivos orais induzindo trombose mesentérica
} Use of oral contraceptives causing mesenteric thrombosis

Josiane L. Simão ${ }^{1}$

Livia C. De Nadai ${ }^{1}$

Patrícia P. Giacon ${ }^{1}$

Márcia Aparecida M. Lopes²

\begin{abstract}
A trombose mesentérica é causa rara de dor abdominal em jovens, sendo responsável por cerca de $5 \%$ a $10 \%$ de todos os eventos de isquemia mesentérica. Contraceptivos hormonais orais têm sido associados a dezenas de casos de trombose mesentérica. Os autores relatam o caso de paciente com diagnóstico de trombose mesentérica após uso de contraceptivos e descrevem a relação entre ambos. M.R.F.S., sexo feminino, 19 anos, branca, deu entrada no Pronto Socorro do Hospital das Clínicas de Marília com quadro de dor abdominal há três dias associada ao uso de cinco comprimidos de anticoncepcional hormonal oral um dia antes de iniciar o quadro. Apresentava-se em regular estado geral, com abdome tenso, enrijecido, com ruídos hidroaéreos hipoativos, doloroso difusamente à palpação, sinal de Jobert e Blumberg positivos. A maioria das causas de trombose mesentérica são devidas a estados pró-trombóticos derivados de desordens da coagulação herdadas ou adquiridas. Portanto, uma vez confirmado este diagnóstico, os pacientes devem ser investigados para trombofilias hereditárias ou adquiridas com testes para deficiência de proteínas Ce S, fator $V$ de Leiden, hiperhomocisteinemia e hemoglobinúria paroxística noturna. Rev. bras. hematol. hemoter. 2008;30(1):75-77.
\end{abstract}

Palavras-chave: Trombose; mesentérica; contraceptivo oral.

\section{Introdução}

A trombose venosa mesentérica é causa rara de dor abdominal em jovens. Contraceptivos orais têm sido considerados um fator associado a dezenas de casos de trombose mesentérica. Foi reconhecida como causa de infarto intestinal há mais de um século por Elliot, mas Warren e Eberhard foram os primeiros a caracterizá-la como causa de isquemia intestinal diferente da oclusão arterial mesentérica. ${ }^{1,2}$

Os autores relatam o caso de uma paciente que desenvolveu um quadro de trombose mesentérica após uso de contraceptivos em altas doses e descrevem a relação entre o uso deste e a patologia diagnosticada.
Relato de caso

M.R.F.S., sexo feminino, 19 anos, branca, solteira, ensino fundamental completo, católica, auxiliar de produção, procedente de Marília. Deu entrada no Pronto Socorro do Hospital das Clínicas de Marília com queixa de dor abdominal há três dias.

Referia dor tipo cólica, intermitente, intensa, sem fatores de alívio, em fossa ilíaca direita há três dias, irradiando para flanco direito e mesogástrio há dois dias. Há um dia tornou-se panabdominal. Associado ao quadro, apresentou náuseas e vômitos, além de um episódio de febre $\left(38,5^{\circ} \mathrm{C}\right)$ há um dia e ausência de evacuação há dois dias. A paciente relatou uso de cinco comprimidos de anti-

${ }^{1}$ Acadêmica da $6^{a}$ série do Curso de Medicina da Faculdade de Medicina de Marília - Marilia-SP.

${ }^{2}$ Docente da Disciplina de Hematologia, responsável pela Residência Médica em Hematologia e Hemoterapia da Faculdade de Medicina de Marília.

Correspondência: Livia Chiosini De Nadai

Rua Marquês de Itu, 545 ap.42

São Paulo-SP - Brasil

Tel.: (11) 3554-7616 / (11) 8308-1836

E-mail: liviadenadai@hotmail.com 
concepcional hormonal oral um dia antes de iniciar o quadro; não soube especificar a medicação. Tinha história pessoal e familiar negativas para fenômenos tromboembólicos.

Ao exame físico apresentava-se em regular estado geral, anictérica, acianótica, afebril, descorada $(+/ 4+)$ desidratada $(+/ 4+)$.

PA: 90 x 60 mmHg; FC: 100 bpm; FR: 20 ipm.

$\mathrm{O}$ abdome apresentava-se tenso, enrijecido, com ruídos hidroaéreos hipoativos, doloroso à palpação difusa e sinal de Blumberg e de Jobert presentes.

Exames complementares: Raio X abdominal: alças distendidas e presença de pneumoperitôneo.

A ultra-sonografia abdominal evidenciou íleo adinâmico, porém descartou processo inflamatório íleocecal.

Hemograma:

- $\mathrm{Hb}=12,8 \mathrm{~g} / \mathrm{dL}(11,5-16,5 \mathrm{~g} / \mathrm{dL})$

- $\mathrm{Ht}=0,39(0,36-0,47)$

- Leucócito:=17,8 x 109\% L (4,0-11 x 10\% L)

- Bastonetes $=14 \times 10^{9} / \mathrm{L}$

- Neutrófilos $=75 \times 10^{9} / \mathrm{L}$, alguns com granulações tóxicas finas

- Linfócitos típicos $=7 \times 10 \%$ L

- Monócitos= 4 × 10\% $/ \mathrm{L}$

- Plaquetas: 297 x 10\%/ L (150-400 x 10\%/ L)

- Urina I: leucócitos: 41.000/mL, demais sem alterações.

- Fibrinogênio: 8,79 g/L (valor de referência: 2-4g/L), sem outras alterações no coagulograma.

A paciente foi submetida a uma laparotomia exploradora, que evidenciou necrose de íleo a cerca de $15 \mathrm{~cm}$ da válvula íleo-cecal, de aproximadamente 1,2 m de extensão.

Laudo do exame anatomopatológico: Infarto hemorrágico, com peritonite fibrino-supurativa. Trombose recente de ramos das veias e artérias mesentéricas.

Após um mês de permanência hospitalar e anticoagulação, a paciente foi encaminhada ao ambulatório de cirurgia vascular onde foi diagnosticada deficiência de proteína C, com valor sérico de 37,3 $\mu / \mathrm{dL}$ (valor de referência: 80- $135 \mu / \mathrm{dL})$.

\section{Discussão}

A trombose mesentérica é responsável por cerca de 5\% a $15 \%$ de todos os eventos de isquemia mesentérica. ${ }^{3} \mathrm{O}$ diagnóstico é sempre demorado; a maioria dos casos é identificada após laparotomia ou necropsia, o que vem sendo modificado com o avanço nas novas técnicas de imagem.

A maioria das causas de trombose mesentérica são estados pró-trombóticos devidos a desordens da coagulação herdadas ou adquiridas, câncer, condições inflamatórias intra-abdominais, pós-operatório, cirrose e hipertensão portal.

O uso de contraceptivos orais é responsável por 9\% a 18\% dos episódios de trombose mesentérica em mulheres jovens. ${ }^{4,5}$
Três influências primárias predispõem à formação de trombo, a chamada Tríade de Virchow: lesão endotelial, estase ou turbulência do fluxo sangüíneo e hipercoagulabilidade sangüínea.

As manifestações clínicas dependem da extensão do trombo, do tamanho dos vasos acometidos e da invasão das camadas da parede intestinal. Quando a isquemia é restrita à mucosa, as manifestações consistem em dor abdominal e diarréia; no caso de isquemia transmural, ocorre necrose com sangramento gastrointestinal, perfuração e peritonite. $^{6}$

A apresentação clínica clássica é dor abdominal tipo cólica em mesogástrio; náusea, vômito, anorexia e diarréia são comuns. Hematêmese, melena ou hematoquezia ocorre em 15\% dos pacientes; ${ }^{7}$ sangramento oculto ocorre em $50 \%$ dos casos. ${ }^{5}$

Cerca de metade dos pacientes têm história pessoal ou familiar de trombose venosa profunda ou embolia pulmonar. ${ }^{8,9}$

A proteína C é um anticoagulante endógeno, dependente da vitamina $\mathrm{K}$, ativada após ligação da trombina ao receptor endotelial trombomodulina e inibe a coagulação clivando os fatores Va e VIIIa. Estas reações são potencializadas pela proteína $\mathrm{S}$, que atua como um cofator não enzimático. Com isso, pode se prever que deficiências dos anticoagulantes naturais, proteínas $\mathrm{C}$ e $\mathrm{S}$, são associadas a um estado de hipercoagulabilidade e a um risco aumentado de tromboembolismo venoso.

A proteína C é originada do cromossomo 2, enquanto a S é originada do cromossomo 3. Deficiências dessas proteínas são consideradas herança autossômica dominante e a heterozigose dessas proteínas pode causar estado de hipercoagulabilidade. ${ }^{10}$ Aproximadamente $50 \%$ dos pacientes com deficiência de proteína C ou S desenvolvem evento trombótico até os 26 anos de idade, e 63\% apresentam recorrência. ${ }^{11}$

Na década de 1980, defeitos genéticos resultando em deficiência de proteína C e S foram reconhecidos pela primeira vez como causas de trombofilia familiar. Deficiências heterozigóticas de proteína $C$ e proteína $S$ são associadas a risco estimado para ocorrência de tromboembolismo venoso aproximadamente dez vezes maior em comparação a indivíduos normais.

Essas deficiências isoladamente são causas bem estabelecidas, porém relativamente raras de trombofilias. ${ }^{12}$

A deficiência de proteína $C$ pode se manifestar de diversas formas: a homozigose resulta em púrpura neonatal fulminante, muitas vezes letal, causada por isquemia de pequenos vasos cutâneos e subcutâneos. Já as manifestações clínicas da heterozigose estão associadas a complicações tromboembólicas venosas, sendo mais freqüentes a trombose venosa profunda de membros inferiores e embolia pulmonar e, mais raramente, trombose das veias mesentérica e cerebral. ${ }^{13}$ 
Uma vez que a trombose venosa mesentérica foi confirmada, os pacientes devem ser investigados para trombofilias hereditárias ou adquiridas com testes para deficiência de proteínas C e S, fator V de Leiden, hiperhomocisteinemia e hemoglobinúria paroxística noturna. Um estudo de medula óssea pode ser realizado na suspeita de doença mieloproliferativa. ${ }^{6}$

O tratamento dos eventos tromboembólicos em pacientes com estados de hipercoagulabilidade primária não difere daqueles em que não há desordens genéticas, estando baseado no uso de trombolíticos nas tromboses maciças e controle com heparina não fracionada ou de baixo peso molecular por pelo menos cinco dias, seguido por profilaxia com warfarin. Devido à meia-vida da proteína $\mathrm{C}$ ser de apenas 6-7 horas, muito menor que a da protrombina e do fator $\mathrm{X}$, um período de hipercoagulabilidade ocorre após o início da terapia com warfarin. Portanto, a heparina deve ser acrescentada nos primeiros dias, até que se atinja a anticoagulação. ${ }^{14}$

A taxa de mortalidade entre os pacientes varia de $20 \%$ a $50 \%$. A sobrevida depende de vários fatores, incluindo idade, presença ou ausência de comorbidades e tempo entre o diagnóstico e a intervenção cirúrgica. Complicações pósoperatórias incluem sepse e síndrome do intestino curto., ${ }^{4,9}$

\begin{abstract}
Mesenteric thrombosis is a rare cause of abdominal pain in the young and is responsible for about $5-10 \%$ of all mesenteric ischemic events. Oral contraceptives are associated to many cases of mesenteric thrombosis. The case of a woman with mesenteric thrombosis after taking a high dose of contraceptives is reported. M.R.F.S., a 19-year-old caucasian woman, arrived in the Emergency Service of the Hospital das Clínicas in Marília reporting abdominal pain over 3 days associated with the use of 5 tablets of oral contraceptives one day earlier. An examination identified the abdominal wall was hardened and tense, with hypoactive bowel sounds, generalized pain on palpation, and Jobert and Blumberg signs. Most causes of mesenteric thrombosis are due to pro-thrombotic conditions caused by acquired or inherited blood coagulation disorders. Thus, when there is suspicion of mesenteric thrombosis, patients should be investigated for acquired and inherited thrombophilia using tests of protein $C$ and $S$, V. Leiden factor, hyperhomocysteinemia and nocturnal paroxysmal hemoglobinuria.. Rev. bras. hematol. hemoter. 2008;30(1):75-77.
\end{abstract}

Key words: Thrombosis; mesenteric; oral contraceptives.

\section{Referências Bibliográficas}

1. Elliot JW. The operative relief of gangrene of intestine due to occlusion of the mesenteric vessels. Ann Surg. 1985;21:9-23.

2. Warren S, Eberhard TP. Mesenteric venous thrombosis. Surg Gynecol Obstet. 1935;61:102-21.

3. Grendell JH, Ockner RK. Mesenteric venous thrombosis. Gastroenterology. 1982;82:358-72.
4. Abdu RA, Zakhour BJ, Dalles DJ. Mesenteric venous thrombosis1911 to 1984 . Surgery. 1987;101:383-8.

5. Harward TR, Green D, Bergan JJ, Rizzo RJ, Yao JS. Mesenteric venous thrombosis. J Vasc Surg. 1989;9:328-33.

6. Kumar S, Sarr MG, Kamath PS. Mesenteric venous thrombosis. N Engl J Med. 2001;345:1683-8.

7. Boley SJ, Kaleya RN, Brandt LJ. Mesenteric venous thrombosis. Surg Clin North Am. 1992;72:183-201.

8. Naitove A, Weisman RE. Primary mesenteric venous thrombosis. Ann of Surgery. 1965;161:516-23.

9. Rhee RY, Gloviczki P, Mendonça CT, et al. Mesenteric venous thrombosis. J Vasc Surg. 1994;20:688-97.

10. Abramson A, Abramson S. Hypercoagulability: clinical assesment and treatment. South Med J. 2001;94(10):1013-20.

11. Pabinger L, Schneider B. Thrombotic risk in hereditary antithrombin III, protein C, or protein S deficiency. A cooperative, retrospective study. Arterioscler Thromb Vasc Biol. 1996;16(6): 742-8.

12. Maffei FHA, Lastoria S, Yoshida WB, Rollo HA. Trombofilias hereditárias. In: Ed. Medsi. Doenças Vasculares Periféricas. Rio de Janeiro: Maracanã; 2002. p.1387-95.

13. Matei D, Brenner B, Marder VJ. Acquired thrombophilic syndromes. Blood Rev. 2001;15:31-48.

14. Agnelli G, Prandoni P, Santamaria MG et al. Warfarin optimal duration Italian Trial Investigatiors: Three months versus one year of oral anticoagulant therapy for idiopathic deep venous thrombosis. N Engl J Med. 2001;345:165-9.

Avaliação: Editor e dois revisores externos

Conflito de interesse: não declarado

Recebido: 14/06/2006

Aceito após modificações: 26/09/2007 